\title{
EVIDENCE FOR THE NAPHTHALENE CATION IN A REGION OF THE INTERSTELLAR MEDIUM WITH ANOMALOUS MICROWAVE EMISSION
}

\author{
S. Iglesias-Groth, A. Manchado, ${ }^{1}$ And D. A. García-Hernández \\ Instituto de Astrofísica de Canarias, C/ Via Láctea s/n, 38200 La Laguna, Spain; sigroth@iac.es \\ J. I. GONZÁLEZ HeRnÁNDEZ ${ }^{2}$ \\ Observatoire de Paris, GEPI, 92195 Meudon Cedex, France \\ AND \\ D. L. LAMBERT \\ W. J. McDonald Observatory, University of Texas, Austin, TX 78712-1083 \\ Received 2008 June 5; accepted 2008 August 8; published 2008 August 28
}

\begin{abstract}
We report high-resolution spectroscopy of the moderately reddened $\left(A_{V}=3\right)$ early-type star Cernis 52 located in a region of the Perseus molecular cloud complex with anomalous microwave emission. In addition to the presence of the most common diffuse interstellar bands (DIBs) we detect two new interstellar or circumstellar bands coincident to within $0.01 \%$ in wavelength with the two strongest bands of the naphthalene cation $\left(\mathrm{C}_{10} \mathrm{H}_{8}^{+}\right)$as measured in gasphase laboratory spectroscopy at low temperatures and find marginal evidence for the third strongest band. Assuming these features are caused by the naphthalene cation, from the measured intensity and available oscillator strengths we find that $0.008 \%$ of the carbon in the cloud could be in the form of this molecule. We expect hydrogen additions to cause hydronaphthalene cations to be abundant in the cloud and to contribute via electric dipole radiation to the anomalous microwave emission. The identification of new interstellar features consistent with transitions of the simplest polycyclic aromatic hydrocarbon adds support to the hypothesis that this type of molecules are the carriers of both diffuse interstellar bands and anomalous microwave emission.
\end{abstract}

Subject headings: ISM: abundances — ISM: lines and bands - ISM: molecules

\section{INTRODUCTION}

Polycyclic aromatic hydrocarbons (PAHs) have been postulated as the natural carriers for (1) the mid-infrared emission features widely observed in the interstellar medium (Puget \& Léger 1989; Allamandola et al. 1985, 1989); (2) the diffuse interstellar bands (DIBs), hundreds of absorption bands in the optical and near-infrared spectra of stars reddened by interstellar material (Léger \& d'Hendecourt 1985; Van der Zwet \& Allamandola 1985) which despite decades of observations remain one of the most enigmatic problems faced by stellar spectroscopists (Herbig 1995; Krełowski et al. 2001; Mulas et al. 2006; Sarre 2006; Hobbs et al. 2008); and (3) the so-called anomalous microwave emission, a diffuse emission detected at high Galactic latitude by several cosmic microwave experiments (Kogut et al. 1996; Leitch et al. 1997; de Oliveira et al. 1999, 2002; Hildebrandt et al. 2007) possibly produced by electric dipole radiation of rapidly spinning carbon-based molecules (Draine \& Lazarian 1998; Iglesias-Groth 2005, 2006).

We decided to explore a possible connection between the carriers of the last two phenomena by performing observations of DIBs toward the Perseus molecular cloud complex where recent observations by Watson et al. (2005) demonstrated the existence of regions of anomalous microwave emission. The microwave interferometer VSA (Very Small Array; Taylor et al. 2003) has recently shown a correlation between the angular structure of the anomalous emission in Perseus and the thermal dust emission measured by the IRAS satellite at angular scales of several arcminutes (Watson et al. 2006). We searched for DIBs in the line of sight of the maximum anomalous microwave emission detected by the VSA by performing high-resolution optical spectroscopy of Cernis $52(\mathrm{BD}+31640)$, a reddened

\footnotetext{
${ }^{1}$ Consejo Superior de Investigaciones Científicas, Spain.

${ }^{2}$ CIFIST Marie Curie Excellence Team.
}

star [excess color $E(B-V)=0.9$; Cernis 1993] located in this line of sight at distance of $240 \mathrm{pc}$, where most of the dust extinction is known to concentrate in the Perseus OB2 dark cloud complex. This is the brightest early-type star in the region of interest. We identify numerous DIBs toward Cernis 52 and report here on the detection of two new bands of likely interstellar origin with wavelengths and strengths consistent with those of the naphthalene cation $\left(\mathrm{C}_{10} \mathrm{H}_{8}^{+}\right)$. Naphthalene is the simplest PAH; its cation has been searched in the interstellar medium (Snow 1992; Krełowski et al. 2001) without a conclusive identification, partly due to the lack of accurate wavelengths for resonance transitions of the cation. Recent gas-phase laboratory spectroscopy at low temperatures provide precise wavelengths, widths, and oscillator strengths (Pino et al. 1999; Romanini et al. 1999; Biennier et al. 2003) and may allow a proper identification of this cation in space.

\section{OBSERVATIONS}

The data were obtained with the SARG spectrograph (Gratton et al. 2001) on the $3.5 \mathrm{~m}$ Telescopio Nazionale Galileo (TNG), the ISIS spectrograph at the $4.2 \mathrm{~m}$ William Herschel Telescope (WHT), both at the Roque de los Muchachos Observatory (La Palma, Spain), and the 2dcoudé cross-dispersed echelle (Tull et al. 1995) on the 2.7 m Harlan J. Smith Telescope at McDonald Observatory (Texas, USA). We observed star Cernis 52 in 2006 November with SARG at resolving power $R=40000$ in the range 5000-9000 $\AA$ and obtained a final combined spectrum with $\mathrm{S} / \mathrm{N} \sim 100$ in the continuum. In addition, star HD 23180 (Cernis 67, also known as $o$ Per) was observed with the same configuration in a nearby line of sight selected with no significant anomalous microwave emission. Fast-rotating early-type stars were also observed for correction of telluric lines. For control of any possible systematic effects further spectroscopic data on Cernis 52 and HD 23180 were 
obtained on 2007 December 28 at WHT using ISIS with grating 1200R, which provided a dispersion of $0.24 \AA$ and $\mathrm{S} / \mathrm{N}$ of 220 in the continuum. Fifteen additional spectra of Cernis 52 where obtained on 2007 August 23, 25, and 26 and 2008 March 1 and 2 with the McDonald 2dcoudé cross-dispersed echelle spectrograph. The spectral range 3740-10160 $\AA$ was recorded with resolving power $R=60000$ and 30000, respectively. These two data sets were independently reduced and properly combined to produce final spectra of $\mathrm{S} / \mathrm{N} \sim 150$ and 300 , respectively. Early-type rapidly rotating hot stars were also observed for correction of any possible telluric absorption in both campaigns. All the spectra were reduced using IRAF, wavelength calibrated with accuracy better than $20 \mathrm{~m} \AA$ pixel $^{-1}$ and coadded after correction for telluric lines. The zero point of the adopted wavelength scale was set by assigning the laboratory wavelengths to the $\mathrm{Na} \mathrm{D}$ interstellar absorption lines recorded in our spectra.

\section{DISCUSSION}

The star Cernis 52 appears embedded in or behind a cloud responsible for significant visible extinction $\left(A_{V}=3\right)$ and absorption bands caused by the intervening interstellar material were expected in the spectrum. We detected the strongest known DIBs (including $\lambda \lambda 5780,5797,5850,6196,6270,6284$, 6376,6379 , and 6614) in the various sets of spectra. The DIBs at 6270,6376 and $6379 \AA$ appear in Cernis 52 with normal strength for the amount of reddening. The DIB at $6284 \AA$ is however remarkably weak. A strong UV field is needed to excite the carrier of this band (Krełowski \& Walker 1987). This strongly argues in favor of a UV shielded environment, a $\zeta$ type cloud (Krełowski \& Sneden 1995), as the source for anomalous microwave emission. The strength of the 5780 DIB is also consistent with such a type of cloud. The DIBs wavelengths show velocity shifts consistent with those of the Na D doublet. A complete study of the DIBs in the line of sight will be presented elsewhere.

Remarkably, we detected first in the TNG spectra and subsequently in each of the other spectra a broad feature near $6707 \AA$, which does not appear in compilations of DIBs (Jenniskens \& Désert 1994; Galazutdinov et al. 2000; Hobbs et al. 2008). In Figure 1 we plot the McDonald and WHT spectra of Cernis 52 showing clear detections of this feature which is not present in either the nearby DIB reference star HD 23180, or the A5 III star HIP 86032 (Allende Prieto et al. 2004). The closest known DIBs are located at 6699.32, 6702.02 and 6729.28 $\AA$ (Hobbs et al. 2008). We detect these three bands in Cernis 52 with equivalent widths of 19, 9, and $10 \mathrm{~m} \AA$, respectively (with an error of $20 \%$ ). They are also found in the comparison star HD 23180 (Galazutdinov et al. 2000). A very narrow absorption feature at $6707.7 \AA$ is also observed in the spectrum of Cernis 52 with an equivalent width of $4 \pm 1 \mathrm{~m} \AA$. This is ascribed to interstellar lithium and appears at a velocity consistent with that of the much stronger $\mathrm{Na}$ I $\mathrm{D}$ doublet interstellar absorption lines measured toward the same star. The Na I doublet shows that the interstellar absorption toward Cernis 52 arises mostly in a discrete individual cloud. There appear to be two competing identifications for the broad $6707 \AA$ feature: a stellar Li I line or an interstellar band from the naphthalene cation.

As shown by the spectrum of HIP 86032 (and other A stars), the $6707 \AA$ region is devoid of stellar lines, the Li I is weak or absent because the $\mathrm{Li}$ is predominantly singly ionized in these atmospheres, and its low abundance may be depleted by mixing. Nonetheless, there are exceptional stars in which the Li I doublet is unexpectedly present; is Cernis 52 such an example? To check

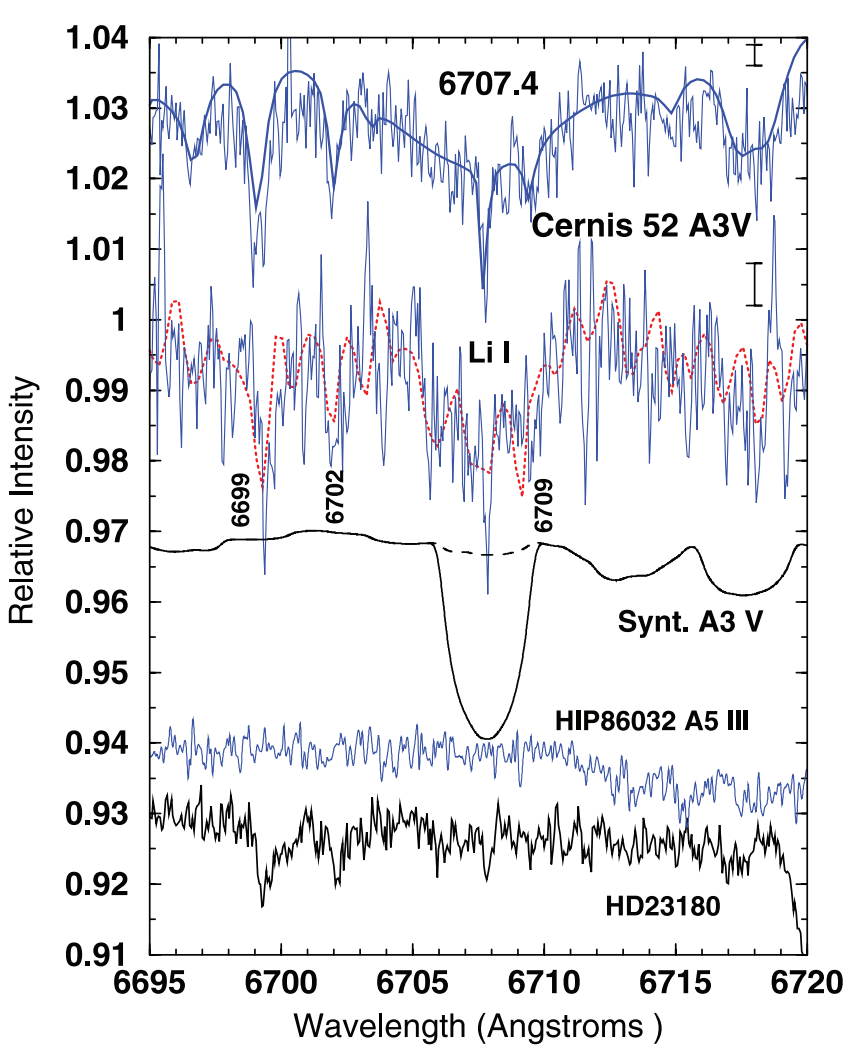

FIG. 1.-Spectra of star Cernis 52 showing three independent detections of the new $\lambda 6707.4$ band. The two spectra at the top (blue thin lines) were obtained with the $2.7 \mathrm{~m}$ telescope at McDonald Observatory in different epochs (see text); $1 \sigma$ error bars are indicated. At the top, we overplot (thick blue line) a fit to the data resulting from a combination of a Lorentzian profile of width $12 \AA$ for this band with suitable profiles for known narrower DIBs and a synthetic photospheric spectrum assuming cosmic $\mathrm{Li}$ abundance. The red line in the second spectrum shows the lower resolution data obtained at the $4.2 \mathrm{~m}$ WHT. The narrow feature at $6707.7 \AA$ is associated with interstellar lithium. The known DIBs at 6699.32, 6702.02, and 6709.43 $\AA$ are marked with wavelength and can be also identified in our spectrum of the hotter reference star HD 23180 (displayed at the bottom). A synthetic spectrum (Synt A3 V) computed using a suitable model atmosphere for Cernis 52 (see text for description) is plotted for two values of $\mathrm{Li}$ abundance $\log [N(\mathrm{Li}) / N(\mathrm{H})]+12=3.3$ and 4.2 , respectively. The synthetic spectra were convolved assuming a rotational velocity of $80 \mathrm{~km} \mathrm{~s}^{-1}$, adequate for the star. The weak broad feature at $6717.7 \AA$ is due to photospheric Ca. Star HIP 86032 (from Allende Prieto et al. 2004) is plotted for comparison.

this possibility, we synthesized the spectrum using the code SYNTHE (Kurucz 2005; Sbordone 2005) for a model atmosphere with an effective temperature of $8500 \mathrm{~K}$, surface gravity of log $g=4.27$, solar metallicity, and a range of $\mathrm{Li}$ abundances. The spectrum was broadened for a rotational velocity of $80 \mathrm{~km} \mathrm{~s}^{-1}$. A $\mathrm{Li}$ abundance of $\log [N(\mathrm{Li}) / N(\mathrm{H})]+12=4.2$ provides the closest fit to the observed feature; see Figure 1 with the stellar radial velocity set by the $\mathrm{Ca}$ I $\lambda 6717$ and other lines. This abundance is 0.9 dex greater than the standard cosmic Li abundance of $\log [N(\mathrm{Li}) / N(\mathrm{H})]+12=3.3$ and such a high Li overabundance is quite exceptional. The sole example among stars of the upper main sequence is apparently a star in the open cluster NGC 6633 (Deliyannis et al. 2002) with a Li abundance of 4.3 but of a lower effective temperature $\left(T_{\text {eff }}=7086 \mathrm{~K}\right)$. The predicted stellar Li I profile is not a satisfactory fit to the observed profile and although we cannot definitively exclude at present the possibility for a remarkable Li overabundance in the star we do not consider this a likely explanation for the $6707 \AA$ feature. Subordinate Li I lines at 6104 and $8126 \AA$ would not be expected to be detectable in our spectra of Cernis 52 . 


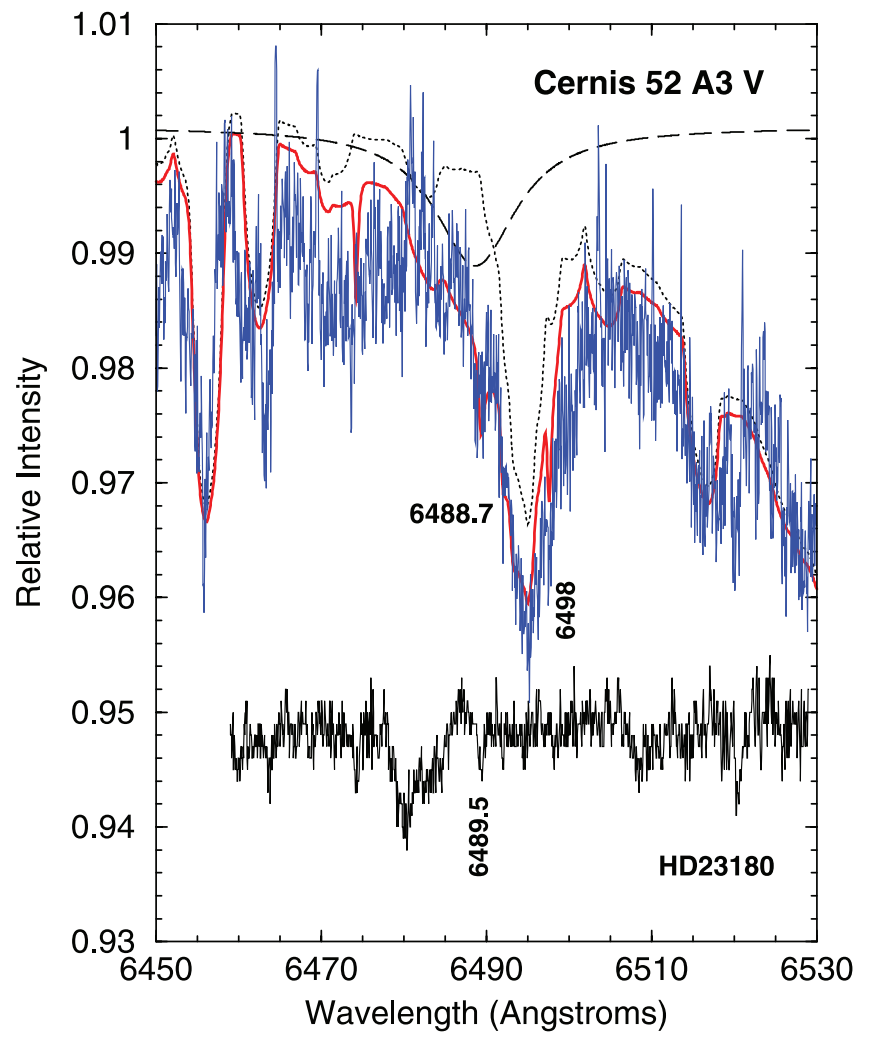

FIG. 2.-Spectrum of the star Cernis 52 (thin blue line) in the region of the second most intense laboratory band of the naphthalene cation. For comparison we plot a synthetic photospheric spectrum (dotted black line) computed using the same stellar model as in the previous figure. The strong absorption at $6495 \AA$ is caused by a combination of photospheric and interstellar absorptions. Superimposed on the blue wing of this feature we find the absorption at $6488.7 \AA$, which we associate with the naphthalene cation. We plot (red line) a combination of the synthetic spectrum with a Lorentzian profile of width $12 \AA$ centered at this wavelength and bands at the positions of known DIBs. At the bottom we display the spectrum of HD 23180 for reference (Galazutdinov et al. 2000). The DIBs at 6489.47 and $6498.08 \AA$ are marked.

Laboratory spectra of the naphthalene cation have been obtained under conditions relevant to the interstellar medium (Pino et al. 1999; Romanini et al. 1999; Biennier et al. 2003). These place the strongest band of the $D_{2} \leftarrow D_{0}$ system at $6707.0 \AA$ with a precision of about $0.5 \AA$ and with a Lorentzian profile with a FWHM of $12 \pm 1 \AA$. In Figure 1 we include a fit that reproduces the observed spectrum (solid line at the top spectrum) using a Lorentzian profile of width $12 \AA$ centered at $6707.4 \AA$ with superimposed profiles for the known DIBs (wavelengths and widths adopted from Hobbs et al. 2008), interstellar lithium, and the photospheric bands. The Lorentzian has an equivalent width of $200 \mathrm{~m} \AA$.

In the search for supporting evidence for this identification of the leading band of the naphthalene cation, we searched for weaker bands. According to laboratory measurements (Biennier et al. 2003), the four strongest naphthalene cation absorption peaks are at $6707.0,6488.9,6125.2$, and $5933.5 \AA$ with a precision of about $0.5 \AA$. Along the series, the intensity decreases as we move to shorter wavelengths, with a ratio of a factor 2 between each two consecutive bands in wavelength. In Figure 2 we plot the spectrum of Cernis 52 in the region of the second most intense transition in the visible spectrum of $\mathrm{C}_{10} \mathrm{H}_{8}^{+}$in the gas phase. This spectral region is dominated by the presence of a very broad band at $6494.2 \AA$ (Jenniskens \& Désert 1994), which results from the combination of pho-

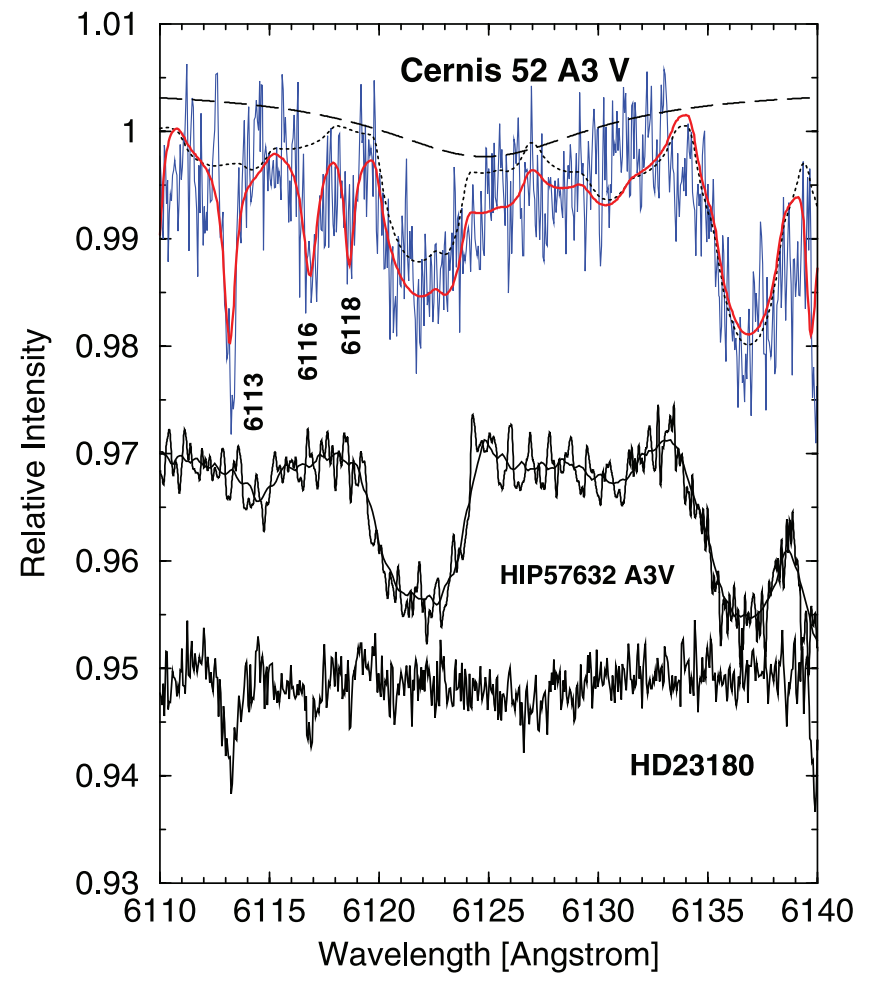

FIG. 3.- Spectrum of star Cernis 52 in the region of the third most intense laboratory band of the naphthalene cation (thin blue line). We plot (thick red line) a combination of a synthetic spectrum (same model as in previous figures) with a Lorentzian of width $12 \AA$ at the expected location for this band and simulation of known DIBs with positions at $6613.18,6616.84$, and $6118.63 \AA$. A comparison is made with the spectrum of a similar faster rotating star HIP 57632 (from Allende Prieto et al. 2004) and with our spectrum of the reference star HD 23180.

tospheric absorptions with a rather broad DIB at $6494.05 \AA$ listed by Hobbs et al. (2008). The location of the stellar continuum in Figure 2 is affected by this broad DIB whose strength is difficult to estimate in Cernis 52 and the blue wing of stellar $\mathrm{H} \alpha$ which makes apparent a progressive decline of the continuum toward the red. We detect a new band at $6488.7 \AA$ in the blue wing of the broad photospheric feature. This band appears partly contaminated by a narrower $(0.9 \AA)$ known DIB at $6489.47 \AA$ (Hobbs et al. 2008). At the top of Figure 2 we plot superimposed on the observed spectrum a combination of a synthetic photospheric spectrum with a Lorentzian of width $12 \AA$ centered at $6488.7 \AA$ and known DIBs in this spectral region simulated using wavelengths and widths from Hobbs et al. (2008). From the area of the Lorentzian we infer an upper limit to the equivalent width of the second naphthalene cation band of $100 \mathrm{~m} \AA$.

The wavelengths and relative strengths of the $\lambda 6488$ and $\lambda 6707$ bands are consistent with those of the first two transitions of the $D_{2} \leftarrow D_{0}$ system of $\mathrm{C}_{10} \mathrm{H}_{8}^{+}$. In Figure 3 we can see a weak feature coincident in position with the third most intense transition of the naphthalene cation at $6125.2 \AA$. Unfortunately, this is superimposed to the red wing of a stellar photospheric feature as shown in the synthetic spectrum plotted in the same figure. We combine the synthetic photospheric spectrum with a Lorentzian of $12 \AA$ (intensity scaled as indicated above) and with a simulation of known DIBs (parameters adopted from Hobbs et al. 2008, as in the previous cases). The good fit of the combined spectrum suggests that indeed the third band of the naphthalene cation could be present with an equivalent 
width of $\sim 50 \mathrm{~m} \AA$. Comparison with similar spectral type stars and the reference star HD 23180 shows no telluric or photospheric bands at this wavelength. Searching for weaker transitions of this cation will require much higher quality data.

Adopting for the oscillator strength of the transition at $6707 \AA$ a value of $f=0.05$ (Pino et al. 1999) and using the equivalent width of the Lorentzian fit, we derive a probable $\mathrm{C}_{10} \mathrm{H}_{8}^{+}$column density of $N_{\mathrm{np}}^{+}=1 \times 10^{13} \mathrm{~cm}^{-2}$ from the Spitzer formula (with an uncertainty of $60 \%$ ). Assuming a ratio $\mathrm{C} / \mathrm{H}=3.7 \times$ $10^{-4}$ and hydrogen column density per unit interstellar absorption $N_{\mathrm{H}} / A_{V}=2 \times 10^{21} \mathrm{~cm}^{-2}$ [with $A_{V}=3 E(B-V)$ ] we calculate that $0.008 \%$ of the total carbon in the intervening cloud could be in the form of naphthalene cations.

In diffuse clouds and dense cloud envelopes that are exposed to the hard interstellar radiation PAHs are expected to be ionized (Wagner et al. 2000; Mattioda et al. 2005). This will be the case of naphthalene with a low ionization potential of $8.1 \mathrm{eV}$. A recent computational study of $\mathrm{H}$-addition reactions to the naphthalene cation shows that most of these reactions have little to no barrier and that hydrogen atoms prefer to join the same ring until it is saturated rather than distribute themselves randomly among the rings (Ricca et al. 2007). Hydrogen additions are expected to occur in energetic regions where the molecule is ionized and there is a large amount of hydrogen available. The hydrogen additions to the naphthalene cation will break the symmetry of the original naphthalene, leading to an effective dipole moment. In the physical conditions of the clouds these molecules are spinning very fast, with rotational frequencies of tens of $\mathrm{GHz}$, and will radiate in the microwave range. We have produced a simple model of the electric dipole emission of these particles to estimate its contribution to the anomalous microwave emission detected in this line of sight. We assume the abundance of naphthalene cations derived above, a dipole moment for the hydrogenated cations comparable to that of the $\mathrm{CH}$ bond $(0.3 \mathrm{D})$, and the typical physical conditions of a Perseus dense molecular cloud (Iglesias-Groth 2005, 2006). We predict that hydrogenated naphthalene cations would contribute in the range (1-5) $\times$ $10^{-21} \mathrm{Jy} \mathrm{cm}^{2} \mathrm{sr}^{-1} \mathrm{H}^{-1}$ at the peak frequency of $80 \mathrm{GHz}$. This is just a very small fraction of the total energy emitted in the microwave range by the cloud but illustrates the role that thermodynamically stable protonated PAHs might play in the anomalous microwave emission.

\section{CONCLUSIONS}

We have recorded the spectrum of star Cernis 52 between 3800 and $10000 \AA$ with resolving power higher than 40000 . In the range 5800-6800 $\AA$, we detect features of likely interstellar origin which are consistent in wavelength and strength ratios with the strongest laboratory gas-phase transitions of the naphthalene cation. These features and other DIBs are detected in the line of sight toward a region of the Perseus molecular complex with previously reported anomalous microwave emission. To the best of our knowledge the new bands have not been previously detected as DIBs. This suggests that the carrier is either not ubiquitous or much less abundant in the general interstellar medium than in the line of sight under investigation. The tentative identification of the cation of the simplest PAH reinforces the hypothesis that this class of molecules could be carriers of the anomalous microwave emission and encourages accurate laboratory gas-phase measurements of other PAHs.

In molecular clouds naphthalene is expected to condense onto refractory dust grains. A large variety of ice mantles can form, but $\mathrm{H}_{2} \mathrm{O}$ will very probably be a dominant species in the ices (Sandford 1996). Recent studies have shown how, under astrophysical conditions, UV radiation on naphthalene in $\mathrm{H}_{2} \mathrm{O}$ ice produces naphthoquinones. These molecules, if further functionalized with a methyl group and a long isoprene chain, play essential roles in biochemical functions (Bernstein et al. 2001). Since quinones and aromatic alcohols are already found in meteoritic material, the detection of naphthalene in molecular clouds shows a possible path for these biogenic compounds from the chemistry of clouds in which new stars form to the prebiotic molecules present in the protoplanetary material.

We thank R. Watson and R. Rebolo for many valuable discussions and B. E. Reddy for help with spectroscopic observations. D. L. L. thanks the Robert A. Welch Foundation of Houston for support. J. I. G. H. acknowledges support from the EU contract MEXT-CT-2004-014265 (CIFIST). This work has been partially founded by project AYA2007-64748 of the Spanish Ministry of Education and Science.

\section{REFERENCES}

Allamandola, L. J., Tielens, A. G. G. M., \& Barker, J. R. 1985, ApJ, 290, L25 1989, ApJS, 71, 733

Allende Prieto, C., et al. 2004, A\&A, 420, 183

Bernstein, M. P., et al. 2001, Meteoritics Planet. Sci., 36, 351

Biennier, L., et al. 2003, J. Chem. Phys., 118, 7863

Cernis, K. 1993, Baltic Astron., 2, 214

Deliyannis, C. P., Steinhauer, A., \& Jeffries, R. D. 2002, ApJ, 577, L39

de Oliveira-Costa, A., et al. 1999, ApJ, 527, L9 2002, ApJ, 567, 363

Draine, B. T., \& Lazarian, A. 1998, ApJ, 494, L19

Galazutdinov, G. A., et al. 2000, PASP, 112, 648

Gratton, R. G., et al. 2001, Experimental Astron., 12, 107

Herbig, G. H. 1995, ARA\&A, 33, 19

Hildebrandt, S. R., et al. 2007, MNRAS, 382, 594

Hobbs, L. M., et al. 2008, ApJ, 680, 1256

Iglesias-Groth, S. 2005, ApJ, 632, L25 . 2006, MNRAS, 368, 1925

Jenniskens, P., \& Désert, F. X. 1994, A\&AS, 106, 39

Kogut, A., et al. 1996, ApJ, 460, 1

Krełowski, J., \& Sneden, C. 1995, in The Diffuse Interstellar Bands, ed. A. G. G. M. Tielens \& T. P. Snow (Dordrecht: Kluwer), 13

Krełowski, J., \& Walker, G. A. H. 1987, ApJ, 312, 860
Krełowski, J., et al. 2001, MNRAS, 328, 810

Kurucz, R. L. 2005, Mem. Soc. Astron. Italiana Suppl., 8, 14

Léger, A., \& d'Hendecourt, L. 1985, A\&A, 146, 81

Leitch, E. M., et al. 1997, ApJ, 486, L23

Mattioda, A. L., Hudgins, D. M., \& Allamandola, L. J. 2005, ApJ, 629, 1188

Mulas, G., Malloci, G., Joblin, J., \& Toublanc, D. 2006, A\&A, 460, 93

Pino, T., Boudin, N., \& Bréchignac, P. 1999, J. Chem. Phys., 111, 7337

Puget, J. L., \& Léger, A. 1989, ARA\&A, 27, 161

Ricca, A., Bakes, E. L. O., \& Bauschlicher, C. W. 2007, ApJ, 659, 858

Romanini, D., et al. 1999, Chem. Phys. Lett., 303, 165

Sandford, S. 1996, in ASP Conf. Ser. 97, Polarimetry of the Interstellar Medium, ed. W. G. Roberge \& D. C. B. Whittet (San Francisco: ASP), 29

Sarre, P. J. 2006, J. Mol. Spectrosc., 238, 1

Sbordone, L. 2005, Mem. Soc. Astron. Italiana Suppl., 8, 61

Snow, T. P. 1992, ApJ, 401, 775

Taylor, A. C., et al. 2003, MNRAS, 341, 1066

Tull, R. G., et al. 1995, PASP, 107, 251

Van der Zwet, G. P., \& Allamandola, L. J. 1985, A\&A, 146, 76

Wagner, D. R., Kim, H. S., \& Saykally, R. J. 2000, ApJ, 545, 854

Watson, R. A., et al. 2005, ApJ, 624, L89

- 2006, in CMB and Physics of the Early Universe, PoS(CMB2006)066 